\title{
Supplement to: Sensitivities of Amazonian clouds to aerosols and updraft speed
}

By Micael A. Cecchini ${ }^{1}$, Luiz A. T. Machado ${ }^{1}$, Meinrat O. Andreae ${ }^{2,12}$, Scot T. Martin ${ }^{3}$, Rachel I. Albrecht ${ }^{4}$, Paulo Artaxo ${ }^{5}$, Henrique M. J. Barbosa ${ }^{5}$, Stephan Borrmann²,6, Daniel Fütterer ${ }^{7}$, Tina Jurkat ${ }^{7}$, Christoph Mahnke ${ }^{2,6}$, Andreas Minikin ${ }^{8}$, Sergej

5 Molleker $^{6}$, Mira L. Pöhlker ${ }^{2}$, Ulrich Pöschl ${ }^{2}$, Daniel Rosenfeld ${ }^{9}$, Christiane Voigt ${ }^{6,7}$, Bernadett Weinzierl ${ }^{7,10}$, Manfred Wendisch ${ }^{11}$

${ }^{1}$ Centro de Previsão de Tempo e Estudos Climáticos, Instituto Nacional de Pesquisas Espaciais, Cachoeira Paulista, Brasil.

${ }^{2}$ Biogeochemistry, Multiphase Chemistry, and Particle Chemistry Departments, Max Planck Institute for Chemistry, P.O. Box 3060, 55020, Mainz, Germany.

$10{ }^{3}$ School of Engineering and Applied Sciences and Department of Earth and Planetary Sciences, Harvard University, Cambridge, Massachusetts, USA.

${ }^{4}$ Departamento de Ciências Atmosféricas, Instituto de Astronomia, Geofísica e Ciências Atmosféricas (IAG), Universidade de São Paulo (USP), Brasil.

${ }^{5}$ Instituto de Física (IF), Universidade de São Paulo (USP), São Paulo, Brasil.

$15{ }^{6}$ Institut für Physik der Atmosphäre (IPA), Johannes Gutenberg-Universität, Mainz, Deutschland.

${ }^{7}$ Institut für Physik der Atmosphäre, Deutsches Zentrum für Luft- und Raumfahrt (DLR), Oberpfaffenhofen, 82234 Wessling, Deutschland.

${ }^{8}$ Flugexperimente, Deutsches Zentrum für Luft- und Raumfahrt (DLR), Oberpfaffenhofen, Deutschland.

${ }^{9}$ Institute of Earth Sciences, The Hebrew University of Jerusalem, Israel.

$20{ }^{10}$ Faculty of Physics, University of Vienna, Boltzmanngasse 5, 1090 Wien, Austria.

${ }^{11}$ Leipziger Institut für Meteorologie (LIM), Universität Leipzig, Stephanstr. 3, 04103 Leipzig, Deutschland.

${ }^{12}$ Scripps Institution of Oceanography. University of California San Diego, La Jolla, CA92093, USA.

Correspondence to: M. A. Cecchini (micael.cecchini@cptec.inpe.br)

\section{DSD profiles for each flight}

25 Figures S1-4 show the individual DSD profiles for each flight considered in this study. It clearly shows the cohesiveness of the aerosol effect on the vertical structure of the warm-phase. Altitudes shown are relative to cloud base. 


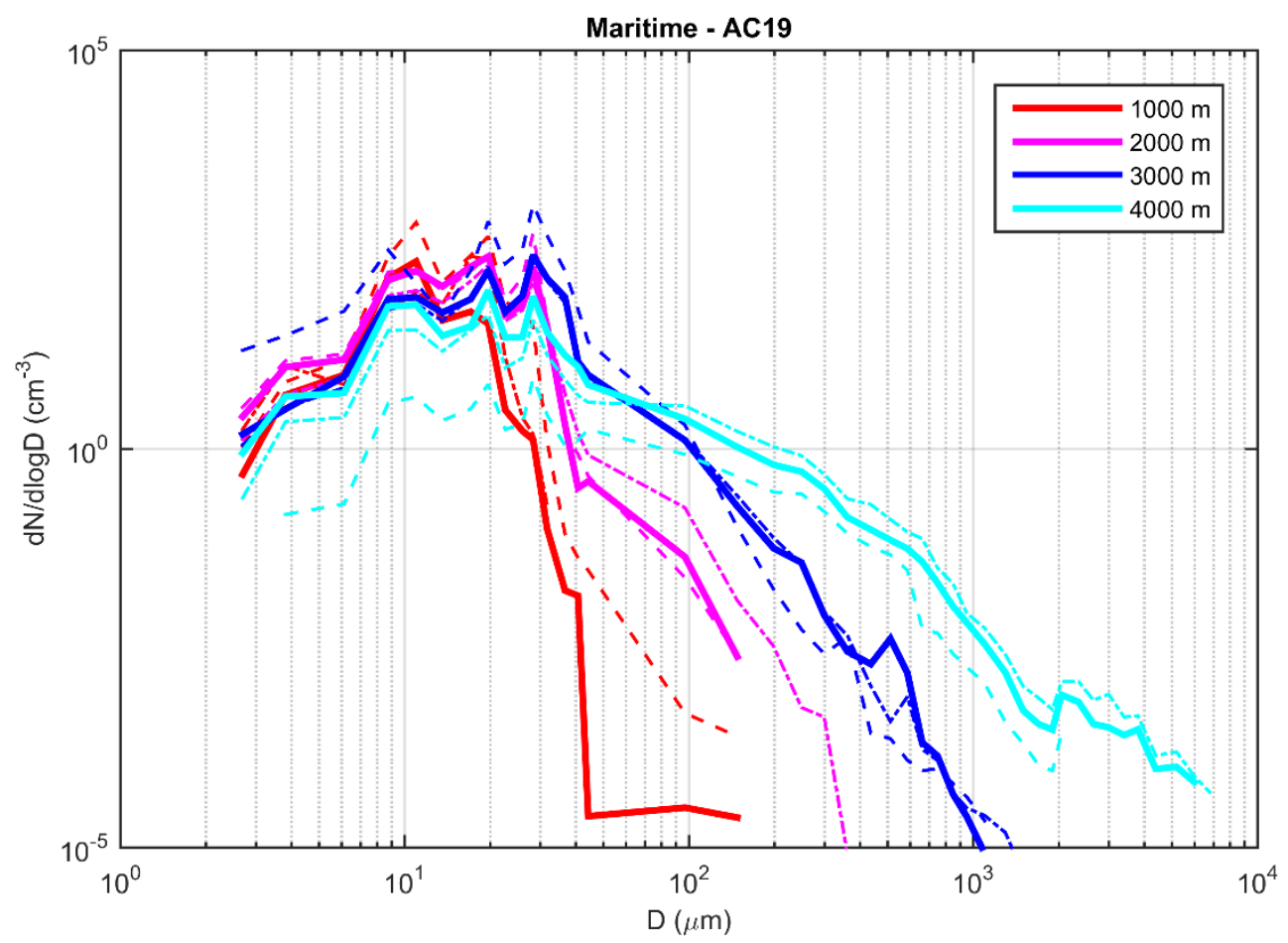

Figure S1. Droplet size distributions as function of altitude above cloud base, aerosol particle number concentration, and vertical wind speed, $W$, for flight AC19. Four 1000-m-thick layers are considered in the vertical, where the legends in the graphs show the respective upper limit of each one. Solid lines represent averaged DSDs for $-1 \mathrm{~m} \mathrm{~s}^{-1} \leq W \leq 1 \mathrm{~m} \mathrm{~s}^{-1}$, i.e., for relatively neutral vertical movements. Dashed lines represent averaged DSDs for the updraft regions where $W>1 \mathrm{~m}$ $\mathrm{s}^{-1}$, and dot-dashed lines represent the downdrafts $\left(W<-1 \mathrm{~m} \mathrm{~s}^{-1}\right)$. 

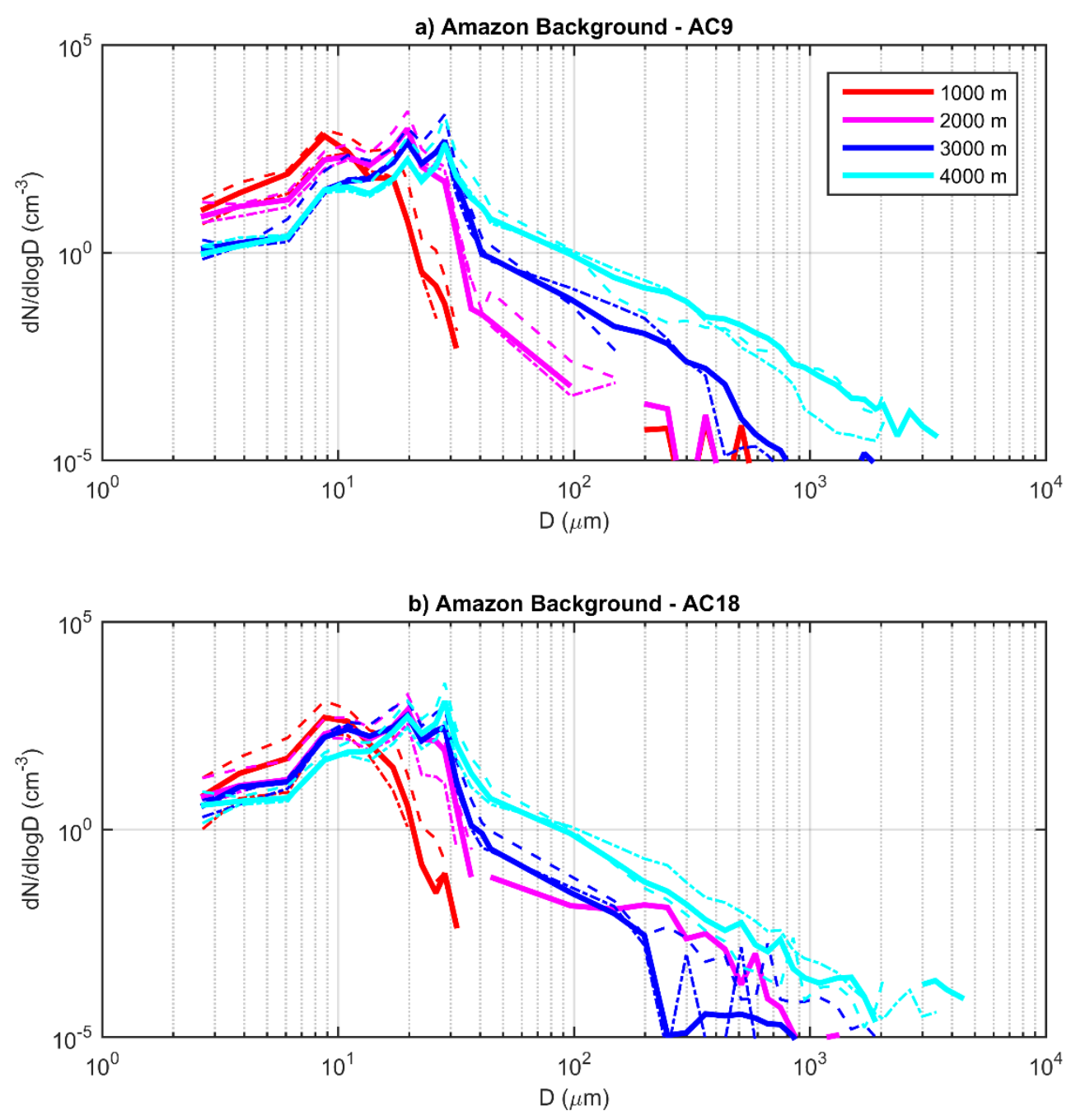

Figure S2. Droplet size distributions as function of altitude above cloud base, aerosol particle number concentration, and vertical wind speed, $W$, for flights a) AC9 and b) AC18. Four 1000-m-thick layers are considered in the vertical, where the legends in the graphs show the respective upper limit of each one. Solid lines represent averaged DSDs for $-1 \mathrm{~m} \mathrm{~s}^{-1} \leq W \leq 1 \mathrm{~m}$

$5 \mathrm{~s}^{-1}$, i.e., for relatively neutral vertical movements. Dashed lines represent averaged DSDs for the updraft regions where $W>1$ $\mathrm{m} \mathrm{s}^{-1}$, and dot-dashed lines represent the downdrafts $\left(W<-1 \mathrm{~m} \mathrm{~s}^{-1}\right)$. 

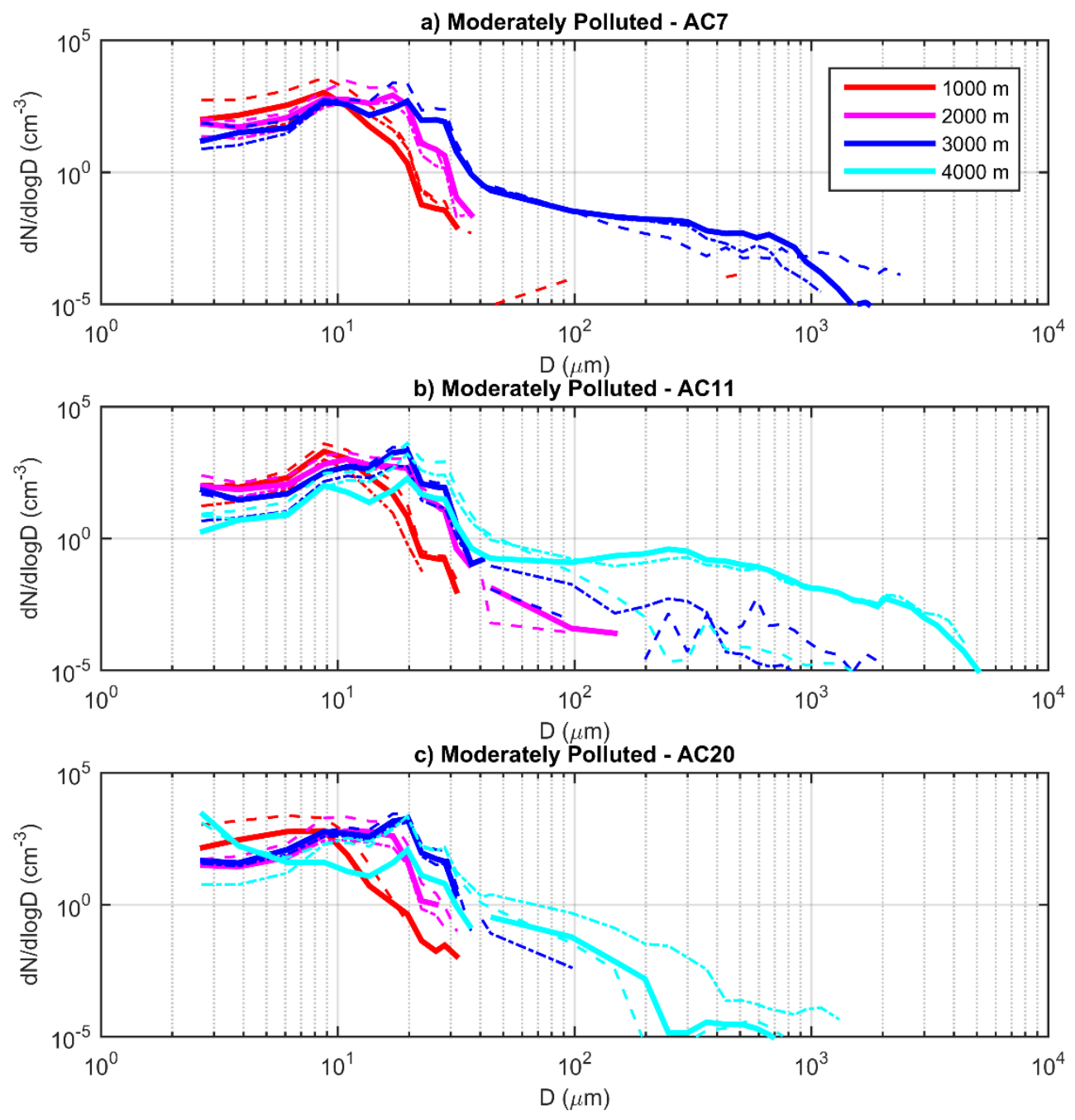

Figure S3. Droplet size distributions as function of altitude above cloud base, aerosol particle number concentration, and vertical wind speed, $W$, for flights a) AC7, b) AC11, and c) AC20. Four 1000-m-thick layers are considered in the vertical, where the legends in the graphs show the respective upper limit of each one. Solid lines represent averaged DSDs for $-1 \mathrm{~m} \mathrm{~s}^{-1}$

$5 \leq W \leq 1 \mathrm{~m} \mathrm{~s}^{-1}$, i.e., for relatively neutral vertical movements. Dashed lines represent averaged DSDs for the updraft regions where $W>1 \mathrm{~m} \mathrm{~s}^{-1}$, and dot-dashed lines represent the downdrafts $\left(W<-1 \mathrm{~m} \mathrm{~s}^{-1}\right)$. 


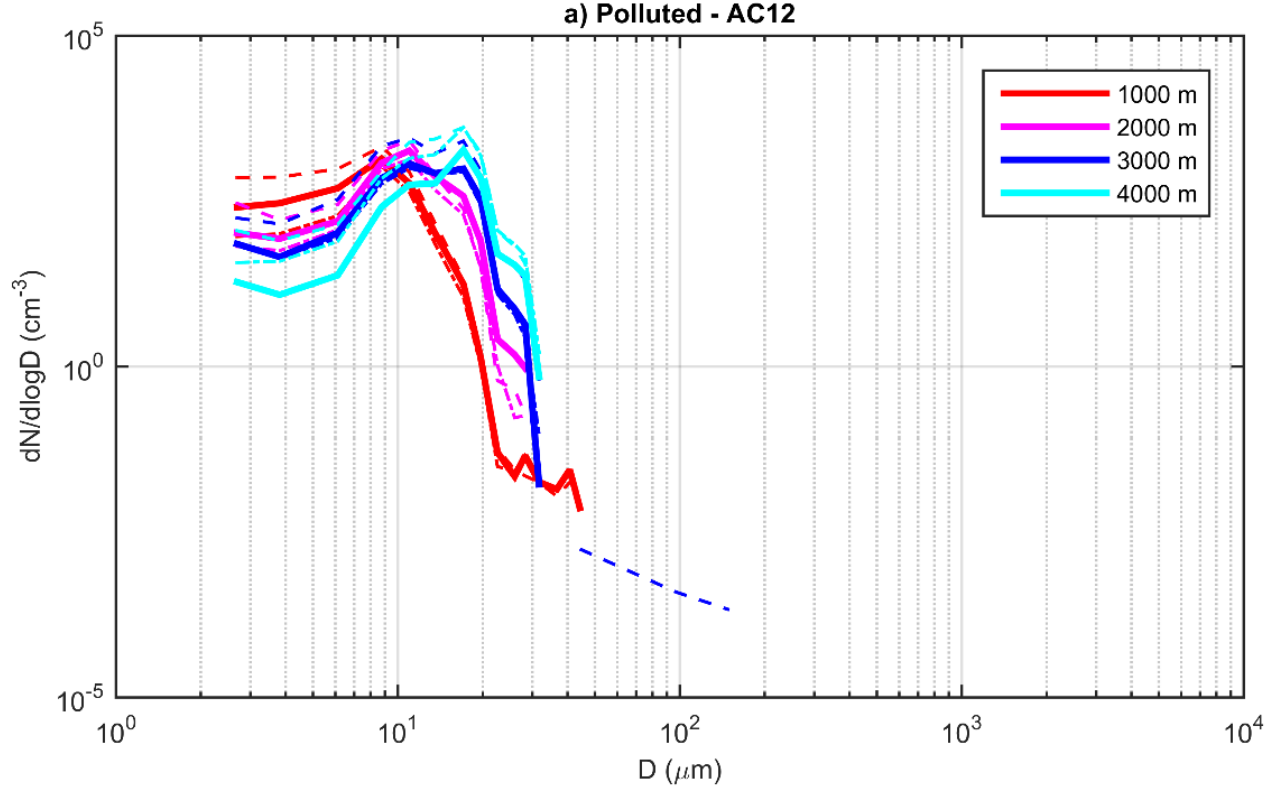

b) Polluted - AC13

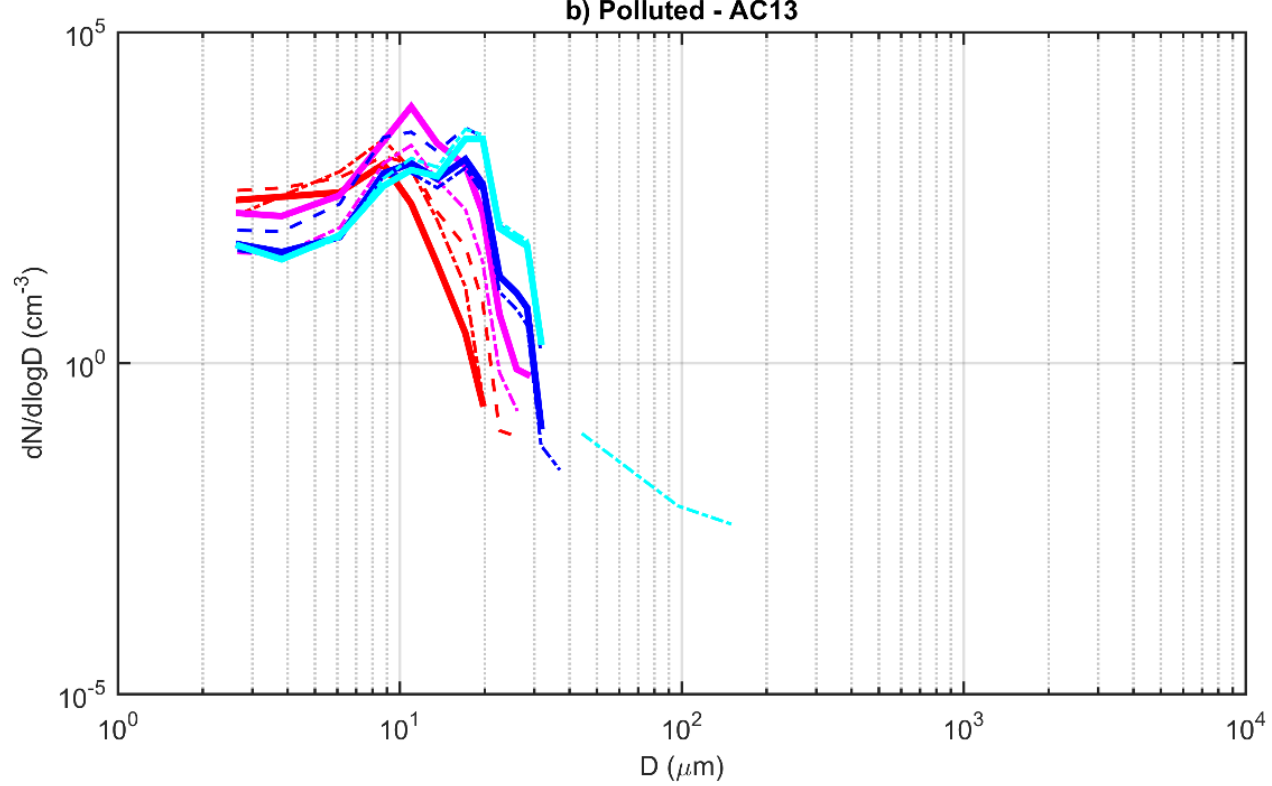

Figure S4. Droplet size distributions as function of altitude above cloud base, aerosol particle number concentration, and vertical wind speed, $W$, for flights a) AC12 and b) AC13. Four 1000-m-thick layers are considered in the vertical, where the legends in the graphs show the respective upper limit of each one. Solid lines represent averaged DSDs for $-1 \mathrm{~m} \mathrm{~s}^{-1} \leq W \leq 1 \mathrm{~m}$

$5 \mathrm{~s}^{-1}$, i.e., for relatively neutral vertical movements. Dashed lines represent averaged DSDs for the updraft regions where $W>1$ $\mathrm{m} \mathrm{s}^{-1}$, and dot-dashed lines represent the downdrafts $\left(W<-1 \mathrm{~m} \mathrm{~s}^{-1}\right)$. 


\section{Sensitivities for individual intervals}

By fixing two dimensions in the 3D matrices and varying the third, we can obtain individual sensitivities in the form of the Equation 1 in the manuscript. As an example, we can fix both $w$ and $H$ and obtain the sensitivities of DSD parameters to varying $N_{a}$. By using the natural logarithm scale and applying a linear fit, we obtain the sensitivity as the angular coefficient

5 and the $\mathrm{R}^{2}$ parameter is a measure of the significance of the relation. By calculating every possible combination, we obtain Tables S1-15 shown below. The amount of $1 \mathrm{~Hz}$ data for each sensitivity are shown in Tables S16-18.

\begin{tabular}{|l|l|l|l|l|l|l|}
\hline$w\left(\mathrm{~m} \mathrm{~s}^{-1}\right) \backslash H(\mathrm{~m})$ & $\mathbf{2 0 0}$ & $\mathbf{5 0 0}$ & $\mathbf{9 5 0}$ & $\mathbf{1 6 2 5}$ & $\mathbf{2 6 3 7 . 5}$ & $\mathbf{4 1 5 6 . 2 5}$ \\
\hline $\mathbf{0 . 5}$ & -0.11 & -0.27 & -0.25 & -0.23 & -0.38 & -0.47 \\
& $\mathrm{R}^{2}=0.85$ & $\mathrm{R}^{2}=0.96$ & $\mathrm{R}^{2}=0.99$ & $\mathrm{R}^{2}=0.94$ & $\mathrm{R}^{2}=0.97$ & $\mathrm{R}^{2}=0.71$ \\
\hline $\mathbf{1}$ & -0.13 & -0.26 & -0.30 & -0.18 & -0.25 & -0.26 \\
& $\mathrm{R}^{2}=0.84$ & $\mathrm{R}^{2}=0.93$ & $\mathrm{R}^{2}=0.99$ & $\mathrm{R}^{2}=0.86$ & $\mathrm{R}^{2}=1.00$ & $\mathrm{R}^{2}=0.96$ \\
\hline $\mathbf{2}$ & -0.16 & -0.26 & -0.28 & -0.17 & -0.31 & -0.16 \\
& $\mathrm{R}^{2}=0.79$ & $\mathrm{R}^{2}=0.98$ & $\mathrm{R}^{2}=0.91$ & $\mathrm{R}^{2}=0.64$ & $\mathrm{R}^{2}=0.98$ & $\mathrm{R}^{2}=0.53$ \\
\hline $\mathbf{4}$ & -0.18 & -0.28 & -0.25 & -0.25 & -0.31 & -0.28 \\
& $\mathrm{R}^{2}=0.82$ & $\mathrm{R}^{2}=0.95$ & $\mathrm{R}^{2}=0.96$ & $\mathrm{R}^{2}=0.95$ & $\mathrm{R}^{2}=0.95$ & $\mathrm{R}^{2}=0.99$ \\
\hline $\mathbf{8}$ & - & - & - & - & -0.26 & -0.33 \\
& & & & & $\mathrm{R}^{2}=0.80$ & $\mathrm{R}^{2}=0.98$ \\
\hline
\end{tabular}

Table S1. sensitivities of $D_{e f f}$ to $N_{a}-S_{D_{e f f}}\left(N_{a}\right)=\left.\frac{\partial \ln D_{e f f}}{\partial \ln N_{a}}\right|_{w, H}$. Intervals upper limits are highlighted in bold letters.

\begin{tabular}{|l|l|l|l|l|l|l|}
\hline$N_{a}\left(\mathrm{~cm}^{-3}\right) \backslash H(\mathrm{~m})$ & $\mathbf{2 0 0}$ & $\mathbf{5 0 0}$ & $\mathbf{9 5 0}$ & $\mathbf{1 6 2 5}$ & $\mathbf{2 6 3 7 . 5}$ & $\mathbf{4 1 5 6 . 2 5}$ \\
\hline \multirow{2}{*}{$\mathbf{5 0 0}$} & 0.020 & 0.049 & 0.048 & -0.018 & 0.032 & - \\
& $\mathrm{R}^{2}=0.63$ & $\mathrm{R}^{2}=0.61$ & $\mathrm{R}^{2}=0.90$ & $\mathrm{R}^{2}=0.034$ & $\mathrm{R}^{2}=0.77$ & \\
\hline \multirow{2}{*}{$\mathbf{1 0 0 0}$} & 0.018 & 0.031 & 0.0072 & 0.046 & 0.0032 & 0.0034 \\
& $\mathrm{R}^{2}=0.17$ & $\mathrm{R}^{2}=0.57$ & $\mathrm{R}^{2}=0.029$ & $\mathrm{R}^{2}=0.71$ & $\mathrm{R}^{2}=0.0040$ & $\mathrm{R}^{2}=0.0010$ \\
\hline \multirow{3}{*}{$\mathbf{3 0 0 0}$} & 0.031 & 0.044 & - & -0.011 & 0.13 & 0.18 \\
& $\mathrm{R}^{2}=0.90$ & $\mathrm{R}^{2}=0.69$ & & $\mathrm{R}^{2}=0.055$ & $\mathrm{R}^{2}=0.93$ & $\mathrm{R}^{2}=0.72$ \\
\hline \multirow{2}{*}{$\mathbf{5 5 0 0}$} & -0.085 & 0.013 & 0.046 & -0.0063 & 0.021 & 0.024 \\
& $\mathrm{R}^{2}=0.97$ & $\mathrm{R}^{2}=0.57$ & $\mathrm{R}^{2}=0.62$ & $\mathrm{R}^{2}=0.23$ & $\mathrm{R}^{2}=0.44$ & $\mathrm{R}^{2}=0.48$ \\
\hline
\end{tabular}

Table S2. sensitivities of $D_{\text {eff }}$ to $w-S_{D_{e f f}}(w)=\left.\frac{\partial \ln D_{e f f}}{\partial \ln w}\right|_{\boldsymbol{N}_{\boldsymbol{a}}, \boldsymbol{H}}$. Intervals upper limits are highlighted in bold letters. 


\begin{tabular}{|l|l|l|l|l|l|}
\hline$N_{a}\left(\mathrm{~cm}^{-3}\right) \backslash w\left(\mathrm{~m} \mathrm{~s}^{-1}\right)$ & $\mathbf{0 . 5}$ & $\mathbf{1}$ & $\mathbf{2}$ & $\mathbf{4}$ & $\mathbf{8}$ \\
\hline \multirow{5}{*}{$\mathbf{0 0 0}$} & 0.33 & 0.27 & 0.31 & 0.32 & - \\
& $\mathrm{R}^{2}=0.98$ & $\mathrm{R}^{2}=0.92$ & $\mathrm{R}^{2}=0.85$ & $\mathrm{R}^{2}=0.92$ & \\
\hline \multirow{2}{*}{$\mathbf{0 0 0}$} & 0.35 & 0.32 & 0.30 & 0.32 & 0.41 \\
& $\mathrm{R}^{2}=0.98$ & $\mathrm{R}^{2}=0.99$ & $\mathrm{R}^{2}=0.95$ & $\mathrm{R}^{2}=1.00$ & $\mathrm{R}^{2}=0.94$ \\
\hline \multirow{3}{*}{$\mathbf{3 0 0 0}$} & 0.14 & 0.23 & 0.28 & 0.26 & 0.27 \\
& $\mathrm{R}^{2}=0.62$ & $\mathrm{R}^{2}=0.90$ & $\mathrm{R}^{2}=0.96$ & $\mathrm{R}^{2}=0.97$ & $\mathrm{R}^{2}=0.96$ \\
\hline \multirow{4}{*}{$\mathbf{4 5 0 0}$} & 0.19 & 0.24 & 0.24 & 0.26 & - \\
& $\mathrm{R}^{2}=0.95$ & $\mathrm{R}^{2}=0.98$ & $\mathrm{R}^{2}=0.99$ & $\mathrm{R}^{2}=0.97$ & \\
\hline
\end{tabular}

Table S3. sensitivities of $D_{\text {eff }}$ to $H-S_{D_{e f f}}(H)=\left.\frac{\partial \ln D_{e f f}}{\partial \ln H}\right|_{N_{a}, \boldsymbol{w}}$. Intervals upper limits are highlighted in bold letters.

\begin{tabular}{|l|l|l|l|l|l|l|}
\hline$w\left(\mathrm{~m} \mathrm{~s}^{-1}\right) \backslash H(\mathrm{~m})$ & $\mathbf{2 0 0}$ & $\mathbf{5 0 0}$ & $\mathbf{9 5 0}$ & $\mathbf{1 6 2 5}$ & $\mathbf{2 6 3 7 . 5}$ & $\mathbf{4 1 5 6 . 2 5}$ \\
\hline \multirow{2}{*}{$\mathbf{* 5}$} & 0.69 & 0.75 & 1.23 & 0.64 & -0.069 & 1.24 \\
& $\mathrm{R}^{2}=0.97$ & $\mathrm{R}^{2}=0.82$ & $\mathrm{R}^{2}=0.89$ & $\mathrm{R}^{2}=0.86$ & $\mathrm{R}^{2}=0.011$ & $\mathrm{R}^{2}=0.83$ \\
\hline $\mathbf{1}$ & 0.67 & 0.79 & 0.90 & 0.87 & 0.70 & 1.11 \\
& $\mathrm{R}^{2}=0.90$ & $\mathrm{R}^{2}=0.87$ & $\mathrm{R}^{2}=1.00$ & $\mathrm{R}^{2}=0.88$ & $\mathrm{R}^{2}=1.00$ & $\mathrm{R}^{2}=0.95$ \\
\hline \multirow{2}{*}{} & 0.72 & 0.89 & 1.049 & 0.87 & 0.90 & 1.40 \\
& $\mathrm{R}^{2}=0.84$ & $\mathrm{R}^{2}=0.98$ & $\mathrm{R}^{2}=0.94$ & $\mathrm{R}^{2}=0.92$ & $\mathrm{R}^{2}=0.92$ & $\mathrm{R}^{2}=0.96$ \\
\hline $\mathbf{4}$ & 0.54 & 0.85 & 0.79 & 0.49 & 0.72 & 1.22 \\
\hline $\mathbf{8}$ & $\mathrm{R}^{2}=0.62$ & $\mathrm{R}^{2}=0.95$ & $\mathrm{R}^{2}=0.99$ & $\mathrm{R}^{2}=0.37$ & $\mathrm{R}^{2}=0.92$ & $\mathrm{R}^{2}=0.98$ \\
\hline & - & - & - & - & 0.94 & 0.83 \\
\hline
\end{tabular}

Table S4. sensitivities of $N_{d}$ to $N_{a}-\boldsymbol{S}_{\boldsymbol{N}_{\boldsymbol{d}}}\left(\boldsymbol{N}_{\boldsymbol{a}}\right)=\left.\frac{\partial \ln \boldsymbol{N}_{\boldsymbol{d}}}{\partial \ln \boldsymbol{N}_{\boldsymbol{a}}}\right|_{\boldsymbol{w}, \boldsymbol{H}}$. Intervals upper limits are highlighted in bold letters. 


\begin{tabular}{|l|l|l|l|l|l|l|}
\hline$N_{a}\left(\mathrm{~cm}^{-3}\right) \backslash H(\mathrm{~m})$ & $\mathbf{2 0 0}$ & $\mathbf{5 0 0}$ & $\mathbf{9 5 0}$ & $\mathbf{1 6 2 5}$ & $\mathbf{2 6 3 7 . 5}$ & $\mathbf{4 1 5 6 . 2 5}$ \\
\hline \multirow{2}{*}{$\mathbf{5 0 0}$} & 0.57 & 0.46 & 0.86 & -0.12 & 0.40 & - \\
& $\mathrm{R}^{2}=1.00$ & $\mathrm{R}^{2}=0.89$ & $\mathrm{R}^{2}=0.97$ & $\mathrm{R}^{2}=0.070$ & $\mathrm{R}^{2}=0.76$ & \\
\hline \multirow{2}{*}{$\mathbf{1 0 0 0}$} & 0.45 & 0.44 & 0.34 & 0.32 & 0.29 & 0.64 \\
& $\mathrm{R}^{2}=0.91$ & $\mathrm{R}^{2}=0.99$ & $\mathrm{R}^{2}=0.47$ & $\mathrm{R}^{2}=0.89$ & $\mathrm{R}^{2}=0.91$ & $\mathrm{R}^{2}=0.89$ \\
\hline \multirow{3}{*}{$\mathbf{3 0 0 0}$} & 0.61 & 0.85 & - & 0.37 & 0.39 & 0.65 \\
& $\mathrm{R}^{2}=0.94$ & $\mathrm{R}^{2}=0.96$ & & $\mathrm{R}^{2}=0.82$ & $\mathrm{R}^{2}=0.95$ & $\mathrm{R}^{2}=0.92$ \\
\hline \multirow{2}{*}{$\mathbf{4 5 0 0}$} & 0.24 & 0.30 & 0.41 & -0.37 & 1.034 & 0.38 \\
& $\mathrm{R}^{2}=0.91$ & $\mathrm{R}^{2}=0.89$ & $\mathrm{R}^{2}=0.67$ & $\mathrm{R}^{2}=0.46$ & $\mathrm{R}^{2}=0.70$ & $\mathrm{R}^{2}=0.90$ \\
\hline
\end{tabular}

Table S5. sensitivities of $N_{d}$ to $w-S_{N_{d}}(w)=\left.\frac{\partial \ln N_{d}}{\partial \ln w}\right|_{N_{a}, H}$. Intervals upper limits are highlighted in bold letters.

\begin{tabular}{|l|l|l|l|l|l|}
\hline$N_{a}\left(\mathrm{~cm}^{-3}\right) \backslash w\left(\mathrm{~m} \mathrm{~s}^{-1}\right)$ & $\mathbf{0 . 5}$ & $\mathbf{l}$ & $\mathbf{2}$ & $\mathbf{4}$ & $\mathbf{8}$ \\
\hline \multirow{5}{*}{$\mathbf{0 0 0}$} & 0.20 & -0.29 & -0.084 & -0.094 & - \\
& $\mathrm{R}^{2}=0.11$ & $\mathrm{R}^{2}=0.97$ & $\mathrm{R}^{2}=0.20$ & $\mathrm{R}^{2}=0.080$ & \\
\hline \multirow{2}{*}{$\mathbf{0 0 0}$} & -0.24 & -0.21 & -0.22 & -0.26 & -0.15 \\
& $\mathrm{R}^{2}=0.36$ & $\mathrm{R}^{2}=0.24$ & $\mathrm{R}^{2}=0.21$ & $\mathrm{R}^{2}=0.54$ & $\mathrm{R}^{2}=0.64$ \\
\hline \multirow{3}{*}{$\mathbf{3 0 0 0}$} & -0.11 & -0.14 & -0.22 & -0.32 & -0.26 \\
& $\mathrm{R}^{2}=0.97$ & $\mathrm{R}^{2}=0.26$ & $\mathrm{R}^{2}=0.94$ & $\mathrm{R}^{2}=0.89$ & $\mathrm{R}^{2}=0.85$ \\
\hline \multirow{2}{*}{$\mathbf{4 5 0 0}$} & -0.26 & 0.068 & 0.075 & 0.081 & - \\
& $\mathrm{R}^{2}=0.094$ & $\mathrm{R}^{2}=0.14$ & $\mathrm{R}^{2}=0.056$ & $\mathrm{R}^{2}=0.022$ & \\
\hline
\end{tabular}

Table S6. sensitivities of $N_{d}$ to $H-\boldsymbol{S}_{N_{d}}(\boldsymbol{H})=\left.\frac{\partial \ln N_{d}}{\partial \ln H}\right|_{\boldsymbol{N}_{a}, \boldsymbol{w}}$. Intervals upper limits are highlighted in bold letters. 


\begin{tabular}{|l|l|l|l|l|l|l|}
\hline$w\left(\mathrm{~m} \mathrm{~s}^{-1}\right) \backslash H(\mathrm{~m})$ & $\mathbf{2 0 0}$ & $\mathbf{5 0 0}$ & $\mathbf{9 5 0}$ & $\mathbf{1 6 2 5}$ & $\mathbf{2 6 3 7 . 5}$ & $\mathbf{4 1 5 6 . 2 5}$ \\
\hline $\mathbf{0 . 5}$ & 0.30 & -0.11 & 0.48 & -0.022 & -1.11 & 0.058 \\
& $\mathrm{R}^{2}=0.97$ & $\mathrm{R}^{2}=0.070$ & $\mathrm{R}^{2}=0.66$ & $\mathrm{R}^{2}=0.013$ & $\mathrm{R}^{2}=0.82$ & $\mathrm{R}^{2}=0.0052$ \\
\hline $\mathbf{1}$ & 0.24 & -0.030 & 0.055 & 0.43 & 0.024 & 0.62 \\
& $\mathrm{R}^{2}=0.40$ & $\mathrm{R}^{2}=0.0072$ & $\mathrm{R}^{2}=0.42$ & $\mathrm{R}^{2}=0.50$ & $\mathrm{R}^{2}=0.12$ & $\mathrm{R}^{2}=0.90$ \\
\hline $\mathbf{2}$ & 0.22 & 0.021 & 0.23 & 0.41 & -0.043 & 0.60 \\
& $\mathrm{R}^{2}=0.26$ & $\mathrm{R}^{2}=0.019$ & $\mathrm{R}^{2}=0.21$ & $\mathrm{R}^{2}=0.34$ & $\mathrm{R}^{2}=0.097$ & $\mathrm{R}^{2}=0.41$ \\
\hline $\mathbf{4}$ & 0.032 & -0.025 & 0.015 & -0.42 & -0.12 & 0.20 \\
\hline $\mathbf{8}$ & $\mathrm{R}^{2}=0.0033$ & $\mathrm{R}^{2}=0.0067$ & $\mathrm{R}^{2}=0.054$ & $\mathrm{R}^{2}=0.25$ & $\mathrm{R}^{2}=0.29$ & $\mathrm{R}^{2}=0.98$ \\
\hline
\end{tabular}

Table S7. sensitivities of $L W C$ to $N_{a}-S_{L W C}\left(N_{a}\right)=\left.\frac{\partial \ln L W C}{\partial \ln N_{\boldsymbol{a}}}\right|_{\boldsymbol{w}, \boldsymbol{H}}$. Intervals upper limits are highlighted in bold letters.

\begin{tabular}{|l|l|l|l|l|l|l|}
\hline$N_{a}\left(\mathrm{~cm}^{-3}\right) \backslash H(\mathrm{~m})$ & $\mathbf{2 0 0}$ & $\mathbf{5 0 0}$ & $\mathbf{9 5 0}$ & $\mathbf{1 6 2 5}$ & $\mathbf{2 6 3 7 . 5}$ & $\mathbf{4 1 5 6 . 2 5}$ \\
\hline \multirow{2}{*}{$\mathbf{5 0 0}$} & 0.62 & 0.60 & 1.024 & 0.060 & 0.34 & - \\
& $\mathrm{R}^{2}=1.00$ & $\mathrm{R}^{2}=0.85$ & $\mathrm{R}^{2}=0.98$ & $\mathrm{R}^{2}=0.0047$ & $\mathrm{R}^{2}=0.91$ & \\
\hline \multirow{2}{*}{$\mathbf{1 0 0 0}$} & 0.50 & 0.42 & 0.37 & 0.42 & 0.31 & 0.69 \\
& $\mathrm{R}^{2}=0.87$ & $\mathrm{R}^{2}=0.90$ & $\mathrm{R}^{2}=0.43$ & $\mathrm{R}^{2}=0.88$ & $\mathrm{R}^{2}=0.85$ & $\mathrm{R}^{2}=0.75$ \\
\hline \multirow{3}{*}{$\mathbf{3 0 0 0}$} & 0.70 & 0.94 & - & 0.33 & 0.70 & 0.89 \\
& $\mathrm{R}^{2}=0.97$ & $\mathrm{R}^{2}=0.94$ & & $\mathrm{R}^{2}=0.72$ & $\mathrm{R}^{2}=0.96$ & $\mathrm{R}^{2}=0.87$ \\
\hline \multirow{2}{*}{$\mathbf{4 5 0 0}$} & 0.10 & 0.33 & 0.53 & -0.47 & 1.00 & 0.42 \\
& $\mathrm{R}^{2}=0.44$ & $\mathrm{R}^{2}=0.84$ & $\mathrm{R}^{2}=0.70$ & $\mathrm{R}^{2}=0.64$ & $\mathrm{R}^{2}=0.66$ & $\mathrm{R}^{2}=0.81$ \\
\hline
\end{tabular}

Table S8. sensitivities of $L W C$ to $w-S_{L W C}(w)=\left.\frac{\partial \ln L W C}{\partial \ln w}\right|_{N_{a}, H}$. Intervals upper limits are highlighted in bold letters. 


\begin{tabular}{|l|l|l|l|l|l|}
\hline$N_{a}\left(\mathrm{~cm}^{-3}\right) \backslash w\left(\mathrm{~m} \mathrm{~s}^{-1}\right)$ & $\mathbf{0 . 5}$ & $\mathbf{1}$ & $\mathbf{2}$ & $\mathbf{4}$ & $\mathbf{8}$ \\
\hline \multirow{5}{*}{$\mathbf{0 0 0}$} & 1.14 & 0.27 & 0.74 & 0.80 & - \\
& $\mathrm{R}^{2}=0.83$ & $\mathrm{R}^{2}=0.45$ & $\mathrm{R}^{2}=0.62$ & $\mathrm{R}^{2}=0.84$ & \\
\hline \multirow{2}{*}{$\mathbf{0 0 0}$} & 0.73 & 0.69 & 0.65 & 0.71 & 1.062 \\
& $\mathrm{R}^{2}=0.92$ & $\mathrm{R}^{2}=0.90$ & $\mathrm{R}^{2}=0.65$ & $\mathrm{R}^{2}=0.92$ & $\mathrm{R}^{2}=0.79$ \\
\hline \multirow{3}{*}{$\mathbf{3 0 0 0}$} & 0.51 & 0.58 & 0.64 & 0.48 & 0.52 \\
& $\mathrm{R}^{2}=0.61$ & $\mathrm{R}^{2}=0.76$ & $\mathrm{R}^{2}=0.95$ & $\mathrm{R}^{2}=0.92$ & $\mathrm{R}^{2}=0.86$ \\
\hline \multirow{4}{*}{$\mathbf{4 5 0 0}$} & 0.36 & 0.77 & 0.70 & 0.76 & - \\
& $\mathrm{R}^{2}=0.16$ & $\mathrm{R}^{2}=0.98$ & $\mathrm{R}^{2}=0.83$ & $\mathrm{R}^{2}=0.62$ & \\
\hline
\end{tabular}

Table S9. sensitivities of $L W C$ to $H-S_{L W C}(H)=\left.\frac{\partial \ln L W C}{\partial \ln H}\right|_{N_{a}, w}$. Intervals upper limits are highlighted in bold letters.

\begin{tabular}{|l|l|l|l|l|l|l|}
\hline$w\left(\mathrm{~m} \mathrm{~s}^{-1}\right) \backslash H(\mathrm{~m})$ & $\mathbf{2 0 0}$ & $\mathbf{5 0 0}$ & $\mathbf{9 5 0}$ & $\mathbf{1 6 2 5}$ & $\mathbf{2 6 3 7 . 5}$ & $\mathbf{4 1 5 6 . 2 5}$ \\
\hline $\mathbf{0 . 5}$ & -0.25 & 0.20 & 0.51 & 0.43 & 0.53 & 0.54 \\
& $\mathrm{R}^{2}=0.50$ & $\mathrm{R}^{2}=0.50$ & $\mathrm{R}^{2}=0.70$ & $\mathrm{R}^{2}=0.74$ & $\mathrm{R}^{2}=0.87$ & $\mathrm{R}^{2}=0.40$ \\
\hline $\mathbf{1}$ & -0.33 & 0.12 & 0.62 & 0.37 & 0.37 & 0.74 \\
& $\mathrm{R}^{2}=0.76$ & $\mathrm{R}^{2}=0.17$ & $\mathrm{R}^{2}=0.87$ & $\mathrm{R}^{2}=0.87$ & $\mathrm{R}^{2}=62$ & $\mathrm{R}^{2}=0.86$ \\
\hline $\mathbf{2}$ & -0.42 & 0.11 & 0.40 & 0.40 & 0.51 & 0.069 \\
& $\mathrm{R}^{2}=0.93$ & $\mathrm{R}^{2}=0.28$ & $\mathrm{R}^{2}=0.91$ & $\mathrm{R}^{2}=0.66$ & $\mathrm{R}^{2}=0.86$ & $\mathrm{R}^{2}=0.13$ \\
\hline $\mathbf{4}$ & -0.54 & -0.15 & 0.062 & 0.29 & 0.56 & 0.14 \\
& $\mathrm{R}^{2}=0.97$ & $\mathrm{R}^{2}=0.39$ & $\mathrm{R}^{2}=0.20$ & $\mathrm{R}^{2}=0.36$ & $\mathrm{R}^{2}=0.88$ & $\mathrm{R}^{2}=0.18$ \\
\hline $\mathbf{8}$ & - & - & - & - & 0.52 & 0.090 \\
& & & & & $\mathrm{R}^{2}=0.99$ & $\mathrm{R}^{2}=0.93$ \\
\hline
\end{tabular}

Table S10. sensitivities of $\Lambda$ to $N_{a}-S_{\Lambda}\left(N_{a}\right)=\left.\frac{\partial \ln \Lambda}{\partial \ln N_{a}}\right|_{w, H}$. Intervals upper limits are highlighted in bold letters. 


\begin{tabular}{|l|l|l|l|l|l|l|}
\hline$N_{a}\left(\mathrm{~cm}^{-3}\right) \backslash H(\mathrm{~m})$ & $\mathbf{2 0 0}$ & $\mathbf{5 0 0}$ & $\mathbf{9 5 0}$ & $\mathbf{1 6 2 5}$ & $\mathbf{2 6 3 7 . 5}$ & $\mathbf{4 1 5 6 . 2 5}$ \\
\hline \multirow{2}{*}{$\mathbf{5 0 0}$} & 0.35 & 0.35 & 0.41 & 0.049 & -0.090 & - \\
& $\mathrm{R}^{2}=0.98$ & $\mathrm{R}^{2}=0.65$ & $\mathrm{R}^{2}=0.66$ & $\mathrm{R}^{2}=0.14$ & $\mathrm{R}^{2}=0.98$ & \\
\hline \multirow{2}{*}{$\mathbf{1 0 0 0}$} & 0.061 & 0.0043 & -0.062 & 0.19 & -0.11 & 0.24 \\
& $\mathrm{R}^{2}=0.24$ & $\mathrm{R}^{2}=0.0037$ & $\mathrm{R}^{2}=0.11$ & $\mathrm{R}^{2}=0.67$ & $\mathrm{R}^{2}=0.75$ & $\mathrm{R}^{2}=0.82$ \\
\hline \multirow{2}{*}{$\mathbf{3 0 0 0}$} & -0.062 & 0.13 & - & 0.015 & -0.14 & -0.15 \\
& $\mathrm{R}^{2}=0.31$ & $\mathrm{R}^{2}=0.55$ & & $\mathrm{R}^{2}=0.045$ & $\mathrm{R}^{2}=0.83$ & $\mathrm{R}^{2}=0.42$ \\
\hline \multirow{2}{*}{$\mathbf{4 5 0 0}$} & -0.0064 & -0.11 & -0.097 & -0.18 & 0.0068 & 0.049 \\
& $\mathrm{R}^{2}=0.13$ & $\mathrm{R}^{2}=0.91$ & $\mathrm{R}^{2}=0.82$ & $\mathrm{R}^{2}=0.23$ & $\mathrm{R}^{2}=0.0089$ & $\mathrm{R}^{2}=0.56$ \\
\hline
\end{tabular}

Table S11. sensitivities of $\Lambda$ to $w-S_{\Lambda}(w)=\left.\frac{\partial \ln \Lambda}{\partial \ln w}\right|_{N_{a}, H}$. Intervals upper limits are highlighted in bold letters.

\begin{tabular}{|c|c|c|c|c|c|}
\hline$N_{a}\left(\mathrm{~cm}^{-3}\right) \backslash w\left(\mathrm{~m} \mathrm{~s}^{-1}\right)$ & 0.5 & 1 & 2 & 4 & 8 \\
\hline 500 & $\begin{array}{l}-0.75 \\
R^{2}=0.96\end{array}$ & $\begin{array}{l}-0.84 \\
R^{2}=0.94\end{array}$ & $\begin{array}{l}-0.94 \\
R^{2}=0.98\end{array}$ & $\begin{array}{l}-1.11 \\
\mathrm{R}^{2}=0.97\end{array}$ & - \\
\hline 1000 & $\begin{array}{l}-0.61 \\
R^{2}=0.98\end{array}$ & $\begin{array}{l}-0.63 \\
R^{2}=0.96\end{array}$ & $\begin{array}{l}-0.47 \\
R^{2}=0.87\end{array}$ & $\begin{array}{l}-0.54 \\
R^{2}=0.86\end{array}$ & $\begin{array}{l}-0.25 \\
R^{2}=0.073\end{array}$ \\
\hline 3000 & $\begin{array}{l}-0.10 \\
R^{2}=0.088\end{array}$ & $\begin{array}{l}-0.17 \\
\mathrm{R}^{2}=0.48\end{array}$ & $\begin{array}{l}-0.25 \\
R^{2}=0.54\end{array}$ & $\begin{array}{l}-0.21 \\
R^{2}=0.34\end{array}$ & $\begin{array}{l}-0.26 \\
R^{2}=0.38\end{array}$ \\
\hline 4500 & $\begin{array}{l}-0.17 \\
R^{2}=0.47\end{array}$ & $\begin{array}{l}-0.15 \\
\mathrm{R}^{2}=0.43\end{array}$ & $\begin{array}{l}-0.15 \\
R^{2}=0.50\end{array}$ & $\begin{array}{l}-0.14 \\
R^{2}=0.62\end{array}$ & - \\
\hline
\end{tabular}

Table S12. sensitivities of $\Lambda$ to $H-S_{\Lambda}(H)=\left.\frac{\partial \ln \Lambda}{\partial \ln H}\right|_{N_{a}, w}$. Intervals upper limits are highlighted in bold letters. 


\begin{tabular}{|l|l|l|l|l|l|l|}
\hline$w\left(\mathrm{~m} \mathrm{~s}^{-1}\right) \backslash H(\mathrm{~m})$ & $\mathbf{2 0 0}$ & $\mathbf{5 0 0}$ & $\mathbf{9 5 0}$ & $\mathbf{1 6 2 5}$ & $\mathbf{2 6 3 7 . 5}$ & $\mathbf{4 1 5 6 . 2 5}$ \\
\hline $\mathbf{0 . 5}$ & 0.17 & 0.013 & -0.17 & -0.12 & -0.097 & -0.097 \\
& $\mathrm{R}^{2}=0.81$ & $\mathrm{R}^{2}=0.014$ & $\mathrm{R}^{2}=0.45$ & $\mathrm{R}^{2}=0.66$ & $\mathrm{R}^{2}=0.56$ & $\mathrm{R}^{2}=0.36$ \\
\hline $\mathbf{1}$ & 0.21 & 0.066 & -0.19 & -0.11 & -0.080 & -0.24 \\
& $\mathrm{R}^{2}=0.93$ & $\mathrm{R}^{2}=0.21$ & $\mathrm{R}^{2}=0.75$ & $\mathrm{R}^{2}=0.80$ & $\mathrm{R}^{2}=38$ & $\mathrm{R}^{2}=0.77$ \\
\hline $\mathbf{2}$ & 0.30 & 0.027 & -0.12 & -0.14 & -0.14 & 0.036 \\
& $\mathrm{R}^{2}=0.95$ & $\mathrm{R}^{2}=0.068$ & $\mathrm{R}^{2}=0.98$ & $\mathrm{R}^{2}=0.50$ & $\mathrm{R}^{2}=0.72$ & $\mathrm{R}^{2}=0.090$ \\
\hline $\mathbf{4}$ & 0.44 & 0.091 & 0.0092 & -0.072 & -0.17 & 0.0031 \\
& $\mathrm{R}^{2}=1.00$ & $\mathrm{R}^{2}=0.24$ & $\mathrm{R}^{2}=0.018$ & $\mathrm{R}^{2}=0.12$ & $\mathrm{R}^{2}=0.70$ & $\mathrm{R}^{2}=0.0012$ \\
\hline $\mathbf{8}$ & - & - & - & - & -0.18 & 0.16 \\
& & & & & $\mathrm{R}^{2}=0.94$ & $\mathrm{R}^{2}=0.95$ \\
\hline
\end{tabular}

Table S13. sensitivities of $\varepsilon$ to $N_{a}-S_{\varepsilon}\left(N_{a}\right)=\left.\frac{\partial \ln \varepsilon}{\partial \ln N_{a}}\right|_{\boldsymbol{w}, \boldsymbol{H}}$. Intervals upper limits are highlighted in bold letters.

\begin{tabular}{|l|l|l|l|l|l|l|}
\hline$N_{a}\left(\mathrm{~cm}^{-3}\right) \backslash H(\mathrm{~m})$ & $\mathbf{2 0 0}$ & $\mathbf{5 0 0}$ & $\mathbf{9 5 0}$ & $\mathbf{1 6 2 5}$ & $\mathbf{2 6 3 7 . 5}$ & $\mathbf{4 1 5 6 . 2 5}$ \\
\hline \multirow{2}{*}{$\mathbf{5 0 0}$} & -0.29 & -0.11 & -0.19 & 0.015 & 0.063 & - \\
& $\mathrm{R}^{2}=0.95$ & $\mathrm{R}^{2}=0.60$ & $\mathrm{R}^{2}=0.80$ & $\mathrm{R}^{2}=0.057$ & $\mathrm{R}^{2}=1.00$ & \\
\hline \multirow{2}{*}{$\mathbf{1 0 0 0}$} & -0.080 & -0.016 & 0.076 & -0.12 & 0.049 & -0.14 \\
& $\mathrm{R}^{2}=0.41$ & $\mathrm{R}^{2}=0.71$ & $\mathrm{R}^{2}=0.34$ & $\mathrm{R}^{2}=0.78$ & $\mathrm{R}^{2}=0.53$ & $\mathrm{R}^{2}=0.86$ \\
\hline \multirow{2}{*}{$\mathbf{3 0 0 0}$} & 0.037 & -0.17 & - & 0.00013 & 0.0024 & -0.035 \\
& $\mathrm{R}^{2}=0.31$ & $\mathrm{R}^{2}=0.76$ & & $\mathrm{R}^{2}=0.000024$ & $\mathrm{R}^{2}=0.0019$ & $\mathrm{R}^{2}=0.22$ \\
\hline \multirow{2}{*}{$\mathbf{4 5 0 0}$} & 0.027 & 0.037 & 0.024 & 0.018 & -0.025 & -0.023 \\
& $\mathrm{R}^{2}=0.51$ & $\mathrm{R}^{2}=0.61$ & $\mathrm{R}^{2}=0.53$ & $\mathrm{R}^{2}=0.30$ & $\mathrm{R}^{2}=0.27$ & $\mathrm{R}^{2}=0.28$ \\
\hline
\end{tabular}

Table S14. sensitivities of $\varepsilon$ to $w-S_{\varepsilon}(w)=\left.\frac{\partial \ln \varepsilon}{\partial \ln w}\right|_{N_{a}, H}$. Intervals upper limits are highlighted in bold letters. 


\begin{tabular}{|c|c|c|c|c|c|}
\hline$N_{a}\left(\mathrm{~cm}^{-3}\right) \backslash w\left(\mathrm{~m} \mathrm{~s}^{-1}\right)$ & 0.5 & $\mathbf{1}$ & 2 & 4 & 8 \\
\hline 500 & $\begin{array}{l}0.22 \\
\mathrm{R}^{2}=0.85\end{array}$ & $\begin{array}{l}0.30 \\
\mathrm{R}^{2}=0.73\end{array}$ & $\begin{array}{l}0.36 \\
\mathrm{R}^{2}=0.94\end{array}$ & $\begin{array}{l}0.48 \\
\mathrm{R}^{2}=0.99\end{array}$ & - \\
\hline 1000 & $\begin{array}{l}0.15 \\
R^{2}=0.79\end{array}$ & $\begin{array}{l}0.16 \\
\mathrm{R}^{2}=0.82\end{array}$ & $\begin{array}{l}0.094 \\
R^{2}=0.63\end{array}$ & $\begin{array}{l}0.16 \\
\mathrm{R}^{2}=0.74\end{array}$ & $\begin{array}{l}-0.16 \\
\mathrm{R}^{2}=0.084\end{array}$ \\
\hline 3000 & $\begin{array}{l}0.0066 \\
\mathrm{R}^{2}=0.0045\end{array}$ & $\begin{array}{l}0.0093 \\
R^{2}=0.030\end{array}$ & $\begin{array}{l}0.017 \\
R^{2}=0.046\end{array}$ & $\begin{array}{l}0.028 \\
\mathrm{R}^{2}=0.032\end{array}$ & $\begin{array}{l}0.010 \\
\mathrm{R}^{2}=0.0024\end{array}$ \\
\hline 4500 & $\begin{array}{l}-0.022 \\
\mathrm{R}^{2}=0.062\end{array}$ & $\begin{array}{l}-0.037 \\
R^{2}=0.20\end{array}$ & $\begin{array}{l}-0.036 \\
R^{2}=0.17\end{array}$ & $\begin{array}{l}-0.046 \\
R^{2}=0.29\end{array}$ & |- \\
\hline
\end{tabular}

Table S15. sensitivities of $\varepsilon$ to $H-S_{\varepsilon}(H)=\left.\frac{\partial \ln \varepsilon}{\partial \ln H}\right|_{N_{a}, w}$. Intervals upper limits are highlighted in bold letters.

\begin{tabular}{|l|l|l|l|l|l|l|}
\hline$w\left(\mathrm{~m} \mathrm{~s}^{-1}\right) \backslash H(\mathrm{~m})$ & $\mathbf{2 0 0}$ & $\mathbf{5 0 0}$ & $\mathbf{9 5 0}$ & $\mathbf{1 6 2 5}$ & $\mathbf{2 6 3 7 . 5}$ & $\mathbf{4 1 5 6 . 2 5}$ \\
\hline $\mathbf{0 . 5}$ & 289 & 89 & 21 & 32 & 36 & 45 \\
\hline $\mathbf{1}$ & 247 & 82 & 20 & 24 & 22 & 45 \\
\hline $\mathbf{2}$ & 223 & 87 & 26 & 34 & 28 & 49 \\
\hline $\mathbf{4}$ & 111 & 47 & 30 & 37 & 29 & 38 \\
\hline $\mathbf{8}$ & 0 & 0 & 0 & 0 & 18 & 27 \\
\hline
\end{tabular}

Table S16. number of $1 \mathrm{~Hz}$ DSD data for the sensitivities to $N_{a}$.

\begin{tabular}{|l|l|l|l|l|l|l|}
\hline$N_{a}\left(\mathrm{~cm}^{-3}\right) \backslash H(\mathrm{~m})$ & $\mathbf{2 0 0}$ & $\mathbf{5 0 0}$ & $\mathbf{9 5 0}$ & $\mathbf{1 6 2 5}$ & $\mathbf{2 6 3 7 . 5}$ & $\mathbf{4 1 5 6 . 2 5}$ \\
\hline $\mathbf{5 0 0}$ & 259 & 84 & 28 & 27 & 11 & 0 \\
\hline $\mathbf{1 0 0 0}$ & 234 & 84 & 38 & 40 & 56 & 81 \\
\hline $\mathbf{3 0 0 0}$ & 265 & 91 & 0 & 61 & 43 & 75 \\
\hline $\mathbf{4 5 0 0}$ & 125 & 55 & 25 & 16 & 23 & 44 \\
\hline
\end{tabular}

5 Table S17. number of $1 \mathrm{~Hz}$ DSD data for the sensitivities to $w$.

\begin{tabular}{|l|l|l|l|l|l|}
\hline$N_{a}\left(\mathrm{~cm}^{-3}\right) \backslash w\left(\mathrm{~m} \mathrm{~s}^{-1}\right)$ & $\mathbf{0 . 5}$ & $\mathbf{1}$ & $\mathbf{2}$ & $\mathbf{4}$ & $\mathbf{8}$ \\
\hline $\mathbf{5 0 0}$ & 169 & 119 & 90 & 35 & 0 \\
\hline $\mathbf{1 0 0 0}$ & 137 & 136 & 146 & 94 & 20 \\
\hline $\mathbf{3 0 0 0}$ & 142 & 100 & 138 & 110 & 51 \\
\hline $\mathbf{4 5 0 0}$ & 64 & 85 & 73 & 53 & 0 \\
\hline
\end{tabular}

Table S18. number of $1 \mathrm{~Hz}$ DSD data for the sensitivities to $H$. 